\title{
Ultrasonografía endoscópica intervencionista. Análisis retrospectivo de 60 procedimientos
}

\author{
M. J. Varas, J. M. Miquel' ${ }^{1}$, R. Abad ${ }^{2}$, J. C. Espinós, M. A. Cañas ${ }^{1}$, R. Fabra ${ }^{1}$ y D. Bargalló \\ Unidad de Ecoendoscopia. Centro Médico Teknon, ${ }^{1}$ Centro Médico Delfos $y^{2}$ Centro Internacional de Medicina \\ Avanzada (CIMA). Barcelona
}

\section{RESUMEN}

Introducción y objetivo: la ultrasonografía endoscópica intervencionista cada día se realiza con más frecuencia debido a que goza de mayores indicaciones. Presentamos nuestra experiencia retrospectiva e inicial (60 procedimientos) en ultrasonografía endoscópica (USE) intervencionista diagnóstica (USE-PAAF) y terapéutica (tumorectomía y mucosectomía guiada por USE).

Pacientes y método: en un grupo de 27 casos, con 10 tumores submucosos (TSM), 2 adenopatías y 15 posibles tumores de páncreas (8 cánceres de páncreas), se practicó USE-PAAF sectorial con 7,5 $\mathrm{MHz}$ con fines diagnósticos previa a la actuación terapéutica (fundamentalmente quirúrgica). Un caso de seudoquiste pancreático fue drenado. En 21 casos con 27 TSM (10 enfermos con 13 carcinoides) se practicó tumorectomía mediante la técnica convencional de polipectomía con asa o asistida con inyección submucosa, y en pocos casos (dos) ligando la lesión con bandas elásticas, previa USE radial con 7,5, 12, o $20 \mathrm{MHz}$. En 6 casos de cáncer superficial gastroesofágico o displasia gástrica se ha practicado resección mucosa endoscópica (RME clásica) previa USE o MS de 7,5 y $20 \mathrm{MHz}$. Se analizaron retrospectivamente 55 pacientes con 60 lesiones, 29 mujeres y 26 varones, con una edad media de 60 años (30-88 años).

Resultados: la precisión diagnóstica $(\mathrm{P})$, sensibilidad (S), especificidad (E), valor predictivo positivo (VPP) y valor predictivo negativo (VPN) de la USE-PAAF fue del 85, 83, 100, 100, y 43\%, respectivamente, al comparar los resultados con la histología de la pieza. La $\mathrm{P}$ fue mayor para las adenopatías (100\%) y tumores pancreáticos (87\%) que para los TSM (80\%). No hubo complicaciones excepto una hemorragia digestiva alta (HDA) (3.7\%) que se trató endoscópicamente y satisfactoriamente en un TSM gástrico. En el grupo de 21 enfermos (10 carcinoides con 13 tumores) se trataron endoscópicamente mediante tumorectomía 27 TSM sin que se registrara ninguna perforación, y tan sólo $2 \operatorname{HDA}(7,4 \%)$ una de ellas autolimitada. sos.

La resección endoscópica fue completa en el 92\% de los ca-

\begin{abstract}
Introduction and objective: interventionist endoscopic ultrasonography is increasingly used because of its growing indications. We present here our retrospective and initial experience $(60$ procedures) with endoscopic ultrasonography (EUS) both for diagnosis (EUS-FNA) and therapy (EUS-guided tumorectomy and mucosectomy).

Patients and method: in a group with 27 cases including 10 submucosal tumors (SMTs), 2 adenopathies, and 15 potential pancreatic tumors (8 pancreatic cancers), a sectorial EUS-FNA at 7.5 $\mathrm{MHz}$ was performed for diagnosis prior to therapy (mainly surgical). A pancreatic pseudocyst was drained. In 21 cases with 27 SMTs (10 patients with 13 carcinoids) a tumorectomy was carried out using the standard loop or assisted polypectomy technique with submucosal injection, and in a few cases (two) using elastic band ligation following a radial EUS at 7.5, 12, or $20 \mathrm{MHz}$. In 6 cases of superficial gastroesophageal cancer or gastric dysplasia an endoscopic mucosal resection (classic EMR) was performed after EUS or MPs at 7.5 and $20 \mathrm{MHz}$. Fifty-five patients with 60 lesions, 29 femaes and 26 males with a mean age of 60 years (30 88 years) were retrospectively analyzed.

Results: diagnostic pecision (P), sensitivity (S), specificity (Sp), positive predictive value (PPV), and negative predictive value (NPV) for EUS-FNA was 85, 83, 100, 100, and 43\%, respectively, when comparing results with specimen histology. $\mathrm{P}$ was higher for adenopathies (100\%) and pancreatic tumors (87\%) than for SMTs (80\%). No complications arose, except for one episode of upper gastrointestinal bleeding (UGIB) (3.7\%) that was endoscopically and satisfactorily treated in a gastric SMT. In the group with 21 patients (10 carcinoids with 13 tumors) 27 SMTs were endoscopically treated by tumorectomy with no perforation and only 2 UGIBs (7.4\%), one of them self-limited, recorded.

Endoscopic resection was complete in $92 \%$ of cases. No complications occurred with classic EMR, and all patients are still alive with no evidence of relapse, either local or metastatic. In this group the rate of complete resections was $100 \%$.
\end{abstract}


Con la técnica de la RME clásica no hubo complicaciones, y todos los pacientes están vivos y sin evidencia de recidiva local o metastásica. En este grupo el porcentaje de resección completa fue del $100 \%$.

Conclusiones: la USE-PAAF es una técnica segura con una buena precisión diagnóstica. La tumorectomía y la mucosectomía asistidas por USE son también técnicas seguras y eficaces en el tratamiento endoscópico de dichos tumores.

Palabras clave: PAAF ecodirigida, tumorectomía y mucosectomía guiada por ecoendoscopia o por ultrasonografía endoscópica. Ultrasonografía endoscópica intervencionista y/o terapéutica.
Conclusions: EUS-FNA is a safe technique with high diagnostic accuracy. EUS-guided tumorectomy and mucosectomy are also safe and effective techniques in the endoscopic management of these tumors.

Key words: EUS-guided FNA, echoendoscopy- or endoscopic ultrasonography-guided tumorectomy and mucosectomy. Interventionist and/or therapeutic endoscopic ultrasonography.

Varas MJ, Miquel JM, Abad R, Espinós JC, Cañas MA, Fabra R, Bargalló D. Ultrasonografía endoscópica intervencionista. Análisis retrospectivo de 60 procedimientos. Rev Esp Enferm Dig 2007; 99: 138-144.

\section{INTRODUCCIÓN}

El objetivo de la ultrasonografía endoscópica (USE) intervencionista puede ser también diagnóstico (USE-PAAF), obteniendo material para su examen histológico, o terapéutico (drenajes, mucosectomía y tumorectomía, etc.) (1).

El objetivo de este trabajo es presentar nuestra experiencia inicial y retrospectiva en USE intervencionista diagnóstica (USE-PAAF) y terapéutica (tumorectomía y mucosectomía guiada por USE) en 60 procedimientos realizados a 55 enfermos.

\section{PACIENTES Y MÉTODOS}

\section{Pacientes y aspectos técnicos}

A veintisiete enfermos con 10 tumores submucosos (TSM), 2 adenopatías metastásicas de cáncer de recto y de páncreas, y 15 posibles tumores en el páncreas, se les practicó USE-PAAF sectorial con 7,5 $\mathrm{MHz}$ (Olympus Europa GF-UCT160-OL5 con un canal de trabajo de 3,7 $\mathrm{mm}$, utilizando agujas Olympus del $22 \mathrm{G}$ ) con fines diagnósticos previa a la actuación terapéutica, fundamentalmente quirúrgica, excepto en un caso que se procedió al drenaje de un gran seudoquiste pancreático.

Se analizaron la precisión diagnóstica (P), sensibilidad (S), especificidad (E), valor predictivo positivo (VPP) y negativo (VPN), mediante las fórmulas habituales.

El citólogo estuvo siempre presente en la sala de exploración.

Veintiún pacientes con 27 TSM (13 carcinoides, 4 abrikosoff, 4 pólipos fibroides, 2 tumores estromales, 1 páncreas ectópico, 1 linfangioma, 1 fibroma y 1 lipoma) fueron estudiados con videoendoscopia, biopsias, y evaluados y estadificados con USE Radial Olympus de 7,5 y $20 \mathrm{MHz}$, con Minisonda (2,6 mm) Fuji-Japón radial y lineal de 12, 15 y 20 $\mathrm{MHz}$, y Minisonda (2,5 mm) Olympus Europa radial y lineal, con sistema DPR de 12 y $20 \mathrm{MHz}$, introducidas a través del canal de trabajo del videoendoscopio $(2,8 \mathrm{~mm})$.
Fundamentalmente se evaluó la profundidad y el tamaño de la lesión, la capa en la que se originaban, las características ecográficas y la presencia de adenopatías.

La técnica de resección endoscópica (tumorectomía) empleada fue la polipectomía con asa, la polipectomía asistida, y dos bandas.

Como mínimo al cabo de un mes se valoró con videoendoscopia y ecoendoscopia la resección completa del tumor, comparándola con la histología de la pieza de resección.

En seis casos de cáncer superficial (4 cánceres superficiales gastroesofágicos, 2 esofágicos y 2 gástricos) o displasia (2 displasias gástricas) se ha practicado RME reglada, con inyección submucosa, succión y polipectomía.

La USE previa se realizó con ecoendoscopio radial Olympus GF-UM 130 de 7,5 y $20 \mathrm{MHz}$, minisonda Fuji radial y lineal de $20 \mathrm{MHz}$, y minisonda Olympus UMDP20-25R con sistema tridimensional DPR.

Fundamentalmente se evaluó el tamaño y la profundidad de la lesión, las capas a las que afectaba, y la presencia de adenopatías.

\section{Aspectos éticos}

Todas las exploraciones se efectuaron bajo consentimiento informado y con sedación (propofol).

En total, se analizaron retrospectivamente 55 enfermos con 60 lesiones recogidas en 4 años, de los cuales 29 eran mujeres y 26 varones, con una edad de 60 años (intervalo: $30-88$ años).

\section{RESULTADOS}

-Resultados de la USE-PAAF: están desglosados en las tablas I y II.

La P, S, E, VPP y VPN fue del 85, 83, 100, 100, y 43\%. La P fue mayor aparentemente en las adenopatías $(100 \%)$ y en el páncreas (87\%), más que en los TSM (80\%) (Fig. 1). 
Tabla I. TSM a los que se le realizó USE-PAAF

\begin{tabular}{|c|c|c|c|c|c|c|c|c|c|}
\hline$N^{\circ}$ & Edad & Sexo & Lugar & Tamaño & Capa & Ecogenicidad & $O D$ & Diagnósti & $O A-P$ \\
\hline 1 & 57 & M & Gástrico & $15 \mathrm{~mm}$ & SM & Ecogénico & Fibrolipoma & P. fibroide & $V_{+}$ \\
\hline 2 & 51 & M & Gástrico & $20-30 \mathrm{~mm}$ & SM & Ecogénico & Fibrolipoma & Lipoma & $V_{+}$ \\
\hline 3 & 53 & M & Gástrico & $15 \mathrm{~mm}$ & SM & Hipoecogénico & GIST o T. & Glómico- & Control \\
\hline 4 & 35 & V & Gástrico & $40-70 \mathrm{~mm}$ & $?$ & Hipoecogénico & Leiomioma & GIST & $V_{+}$ \\
\hline 5 & 79 & $M$ & Gástrico & $51 \mathrm{~mm}$ & SM & Hipoecogénico & GIST?-HDA & $?$ & $\mathrm{~F}-$ \\
\hline 6 & 88 & $M$ & Gástrico & $25-50 \mathrm{~mm}$ & MP/SM & Hipoecogénico & GIST O T. M. & GIST & $V_{+}$ \\
\hline 7 & 64 & V & Gástrico & 100 mm & $?$ & Hipoecogénico & GIST o T.M. & GIST & $V_{+}$ \\
\hline 8 & 47 & V & Esofágico & $25 \mathrm{~mm}$ & SM/MP & Hipoecogénico & GIST o T.M. & T.M. & $V_{+}$ \\
\hline 9 & 45 & $M$ & Gástrico & $20 \mathrm{~mm}$ & SM & Hipoecogénico & T. estromal & GIST & $V_{+}$ \\
\hline 10 & 64 & $M$ & Gástrico & $15 \mathrm{~mm}$ & SM & Ecogénico & Fibrolipoma & $?$ & F- \\
\hline
\end{tabular}

OD: orientación diagnóstica por USE, SM: submucosa, MP: muscular propia. V+: verdadero positivo, F-: falso negativo; Media de 2 pases. P: $80 \%$.

Tabla II. Casos pancreáticos a los que se le realizó USE-PAAF

\begin{tabular}{|c|c|c|c|c|c|c|c|}
\hline$N^{\circ}$ & Edad & Sexo & Tamaño & O. diagnóstica & Diagnóstico & $A-P$ & \\
\hline 1 & 47 & M & $9 \mathrm{~mm}$ & TNE & Céls. duodenales & & $\mathrm{F}-$ \\
\hline 2 & 44 & V & - & PCC-CP? & No atipias & PCC & $V-$ \\
\hline 3 & 70 & V & $31-48 \mathrm{~mm}$ & PC-CP & Atipias & $\mathrm{CP}$ & $V+$ \\
\hline 4 & 66 & V & $20-30 \mathrm{~mm}$ & $C P$ & & $C P$ & $V+$ \\
\hline 5 & 77 & M & $20 \mathrm{~mm}$ & $C P$ & & $C P$ & $V+$ \\
\hline 6 & 57 & V & $20-30 \mathrm{~mm}$ & $\mathrm{CP}$ & & $C P$ & $V+$ \\
\hline 7 & 77 & M & $20-30 \mathrm{~mm}$ & $C P$ & & $C P$ & $V+$ \\
\hline 8 & 59 & V & $40 \mathrm{~mm}$ & $\mathrm{CP}$ & & $C P$ & $V+$ \\
\hline 9 & 83 & M & $20 \mathrm{~mm}$ & $P C-C P ?$ & Céls. duodenales & & V - \\
\hline 10 & 64 & V & - & ED o TMPI & & TMPI & $V+$ \\
\hline 11 & 70 & M & $30-40 \mathrm{~mm}$ & $C P$ & & $C P$ & $V+$ \\
\hline 12 & 71 & M & $20 \mathrm{~mm}$ & PC-CP ? & & $C P$ & $V+$ \\
\hline 13 & 50 & M & $5,5-10,2 \mathrm{~mm}$ & TNE & & $\mathbb{I P}$ & $V_{+}$ \\
\hline 14 & 55 & V & $10 \mathrm{~mm}$ & TNE & & GP & $\mathrm{F}-$ \\
\hline 15 & 68 & V & $\begin{array}{l}30-50 \mathrm{~mm} \\
9-50 \mathrm{~mm}\end{array}$ & Cistoadenoma & & & V- \\
\hline
\end{tabular}

TNE: tumor neuroendocrino; PC: pancreatitis crónica; CP: cáncer de páncreas; ED: ectasia ductal; TMPI: tumor mucinoso papilar intraductal; Media 4 pases. P: 87\%. S: 83\%. E: $100 \%$. P en las adenopatías (2 casos): $100 \%$.

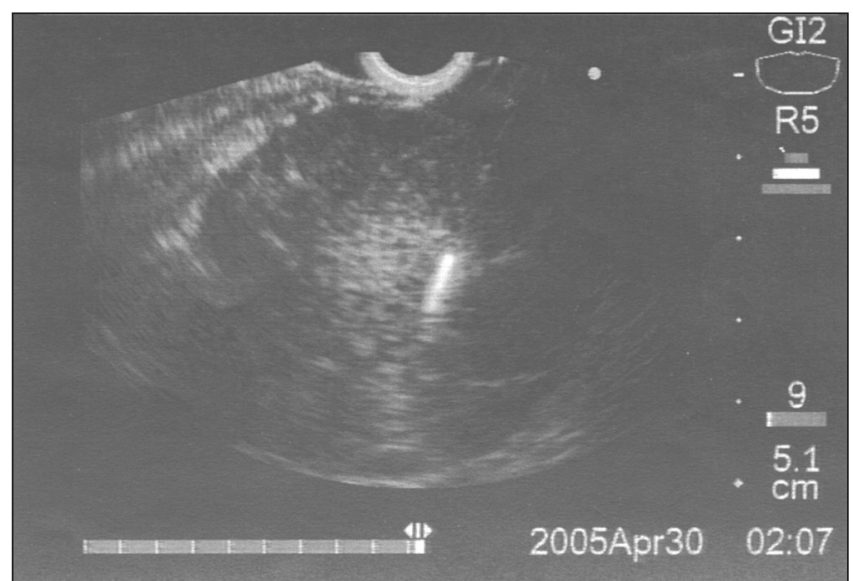

Fig. 1. PAAF-USE sectorial de un GIST gástrico.

En los 27 casos en que se practicó USE-PAAF sólo hubo una complicación, una hemorragia $(3,7 \%)$ que se trató endoscópicamente y satisfactoriamente con inyec- ción de adrenalina en una paciente con un TSM gástrico de $51 \mathrm{~mm}^{\left(\text {caso }^{\circ}\right.}$ 5) (Tabla I).

-Resultados de la tumorectomía:

En los 27 TSM en que se practicó la tumorectomía no hubo ninguna perforación ( $0 \%$ ), y sólo dos hemorragias $(7,4 \%)$, una autolimitada $(3,7 \%)$. La resección endoscópica fue completa en el $92 \%$ de los casos (Fig. 2).

-Resultados de la mucosectomía:

Con la técnica de la RME clásica tampoco hubo complicaciones, ni recidiva local o metastásica. La resección fue completa en el 100\% de los casos (Fig. 3).

\section{DISCUSIÓN}

La USE primordialmente se utiliza para la evaluación de lesiones submucosas y para la estadificación del cáncer del tracto digestivo y del páncreas. En algunas situaciones se requiere obtener material histológico para reafirmar el diagnóstico de sospecha, efectuándose PAAF 

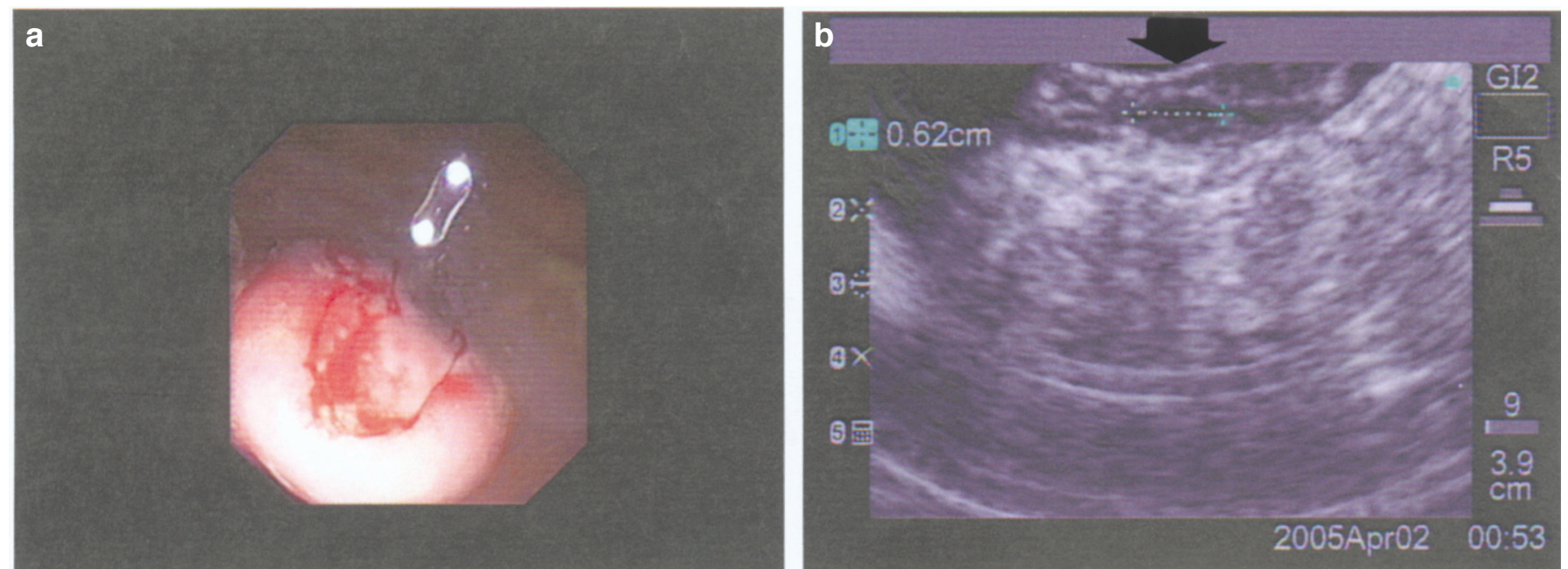

Fig. 2. Tumorectomía o polipectomía de un carcinoide rectal (a) en función de la USE sectorial con 7.5 MHz (b).
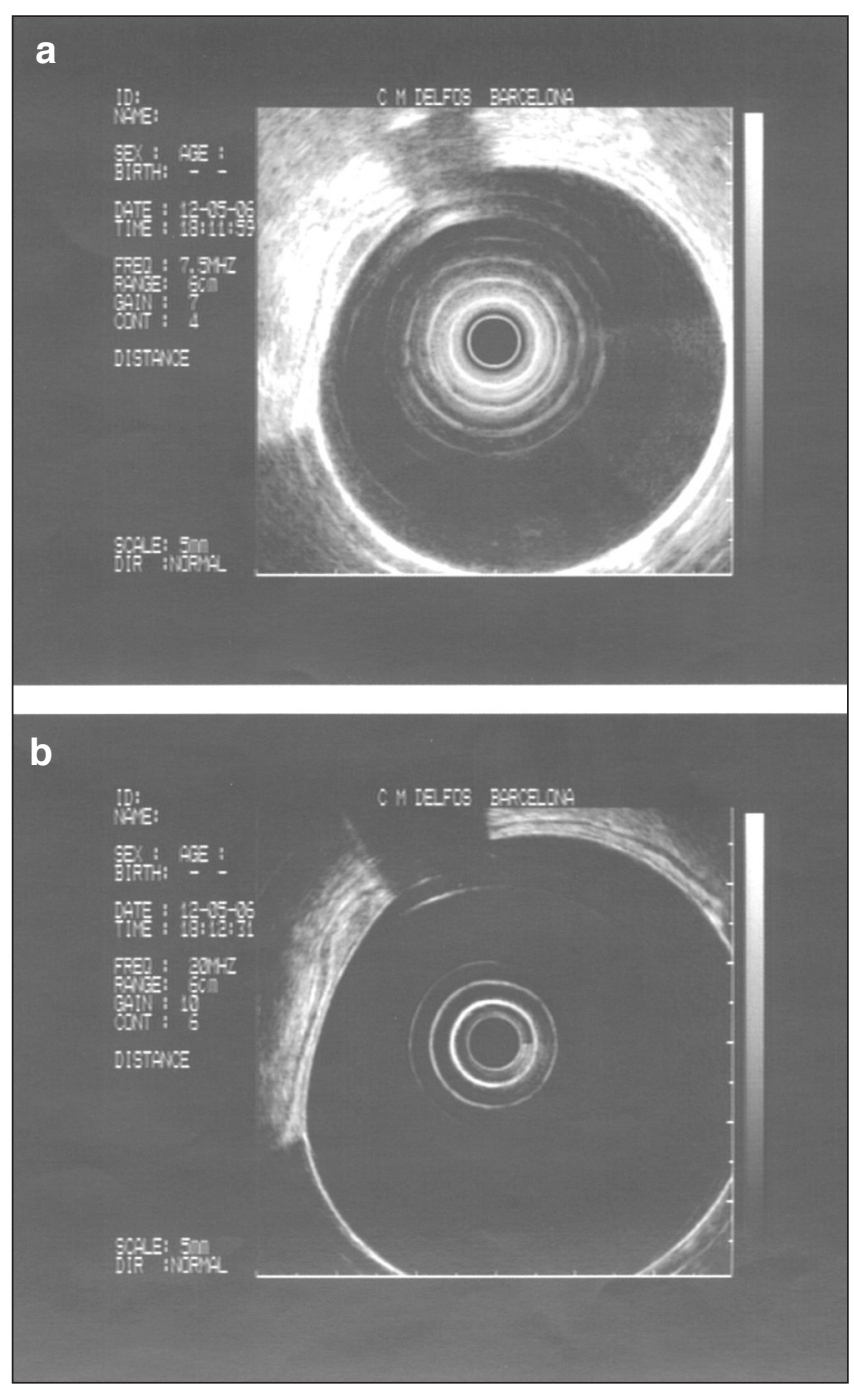

Fig. 3. T1 mucoso NO gástrico candidato a mucosectomía. USE radial con 7.5 (a) y $20 \mathrm{MHz}$ (b). ecodirigida (USE-PAAF) (2-4). Con ello se consigue una estadificación más precisa en las neoplasias del aparato digestivo (5).

En la mayoría de los TSM la endoscopia tiene una precisión inferior al $40 \%$ para identificar la causa de las lesiones submucosas, mientras que la ecoendoscopia tiene una sensibilidad del $94 \%$ (6). La biopsia endoscópica no es útil para el diagnóstico, siendo mejorada por la macrobiopsia (90\%), la cual no está exenta de complicaciones. El rendimiento en la obtención de material histológico mediante USE-PAAF en el caso de los TSM se sitúa alrededor del 60-70\% $(7,8)$ con escasas complicaciones, es inferior al de las masas extrínsecas a la pared digestiva (64 versus 93\%) (9). En los últimos trabajos dicho porcentaje ha aumentado notablemente hasta más del 90\% (9-16) y el diagnóstico ha mejorado con la introducción de la inmunocitoquímica (17).

Nuestros resultados (P: 80\%) han sido similares a los trabajos más recientes (Tabla I).

En las adenopatías metastásicas la precisión y sensibilidad de la USE-PAAF se sitúa alrededor del 90\% (2,7,8, 18-21), con una especificidad que puede llegar a ser del 100\% (20), estando exenta de complicaciones (0\%) (Tablas II y III).

Estos últimos porcentajes se reproducen y aumentan al puncionar las lesiones pancreáticas $(2,7,8)$, si bien es importante disponer de un citólogo presente en la sala de exploración (22). Los porcentajes pueden disminuir en presencia de pancreatitis crónica (del 85 al 54\%) en un estudio sobre 207 pacientes consecutivos (23).

La sensibilidad, especificidad, VPP y VPN de la USEPAAF para el diagnóstico histológico de lesiones sólidas pancreáticas es de 95, 100, 100 y $85 \%$ respectivamente, valores no alcanzados por ninguna otra técnica diagnóstica (24), sin que el tamaño de la tumoración afecte a la precisión de la USE-PAAF (Tabla III).

La incidencia de complicaciones es baja: 1,09\%; 0,5\% para las lesiones sólidas pero las lesiones quísticas, sobre todo pancreáticas, tienen un riesgo de hemorragia y/o per- 
Tabla III. PAAF-USE. Algunos resultados de la literatura

\begin{tabular}{|c|c|c|c|c|c|}
\hline Autor-año & $N^{0}$ & C & Pre & $S$ & $E$ \\
\hline \multicolumn{6}{|l|}{ PAAF-TSM: } \\
\hline Giovannini (1995) (7) & 7 & 0 & 71 & 60 & $100 \%$ \\
\hline Wiersema (1997) (8) & 115 & & 67 & 61 & $79 \%$ \\
\hline Gress (1997) (10) & 27 & & 81 & & \\
\hline \multirow[t]{2}{*}{ Matsui (1998) (11) } & 22 & & 82 & & \\
\hline & 15 & & 93 & & \\
\hline Gu (2001) (12) & 12 & & & & \\
\hline Ando (2002) (13) & 23 & & 91 & & \\
\hline Arantes (2004) (16) & 10 & & 80 & & \\
\hline Varas (2006) & 10 & $1 \mathrm{c}$ & 80 & & \\
\hline \multicolumn{6}{|l|}{ PAAF-ADENO: } \\
\hline Giovannini (1995) (7) & 50 & 0 & 83 & 81 & $100 \%$ \\
\hline Wiersema (1997) (8) & 192 & & 92 & 92 & $93 \%$ \\
\hline Harada (1997) (18) & 211 & & 87 & 80 & $94 \%$ \\
\hline Williams (1999) (19) & 160 & & 89 & 85 & $100 \%$ \\
\hline$X(2000)(2)$ & 793 & 0 & 87 & 87 & $96,5 \%$ \\
\hline Fritscher-R (2000) (20) & 153 & & 94 & 90 & $100 \%$ \\
\hline Chen (2004) (21) & 183 & & 99 & 98 & $100 \%$ \\
\hline \multicolumn{6}{|l|}{ PAAF-TP: } \\
\hline Chang (1994) (9) & 24 & 0 & 94 & 91 & $100 \%$ \\
\hline Giovannini (1995) (7) & 43 & 0 & 79 & 75 & $100 \%$ \\
\hline Chang (1996) (22) & 164 & 2 & 85 & 83 & $90 \%$ \\
\hline Wiersema (1997) (8) & 124 & 1,9 & 88 & 86 & $94 \%$ \\
\hline Gress (1997) (10) & 121 & 3,3 & 85 & 80 & $100 \%$ \\
\hline Williams (1999) (19) & 144 & 0 & 85 & 82 & $100 \%$ \\
\hline$X(2000)(2)$ & 986 & 1,5 & 88 & 86 & $97 \%$ \\
\hline Fritscher-R (2002) (23) & 207 & - & & 85 & \\
\hline Eloubeidi (2003) (24) & 101 & - & - & 95 & $100 \%$ \\
\hline Varas (2006) & 15 & 0 & 87 & 83 & $100 \%$ \\
\hline
\end{tabular}

C: complicaciones; Pre: precisión; S: sensibilidad; E: especificidad.

foración del 14\% (8) en la serie más larga publicada. Otros autores refieren poquísimas complicaciones, 0 y $0,3 \%$ ( 1 caso de sepsis) respectivamente $(7,19)$.

En un trabajo más reciente el porcentaje de complicaciones se sitúa en el 1,24\% (25), 4/322, tres pancreatitis al puncionar lesiones quísticas pancreáticas y una neumonía por aspiración al puncionar un GIST. La USE-PAAF no se asocia con bacteriemia, sepsis, existiendo un bajo riesgo de complicaciones infecciosas (26).

Durante 13 años de experiencia las complicaciones de la USE se cifran en el 0,093 y en el 2,2\% (5/224) para la USE-PAAF (27). La mayoría, 4, se produjeron al puncionar tumores pancreáticos y 1 al puncionar un tumor mixto del mediastino.

La mayor complicación de la PAAF de los TMS es la hemorragia. En nuestra corta serie ha sido la única complicación (1 caso) relacionada con la técnica (Tabla I).

La alternativa a la cirugía abierta o laparoscópica de los TSM lo constituye la tumorectomía endoscópica guiada con USE (28-39), ya que para realizarla con seguridad se requiere que los tumores sean menores de $2 \mathrm{~cm} \mathrm{y}$ estén localizados en las tres primeras capas. Existe ya una experiencia publicada de más de 350 casos (Tabla IV), sin complicaciones importantes, prácticamente un $0 \%$ de perforación y aproximadamente un $10 \%$ de hemorragia,
Tabla IV. Tumorectomía asistida por USE. Revisión de la literatura

\begin{tabular}{|c|c|c|c|c|c|c|}
\hline Autor & $N^{0}$ & Perf. & $H D A$ & $R C$ & $N R$ & \\
\hline $\begin{array}{l}\text { Kawamoto (1997) } \\
\text { (28) }\end{array}$ & $45 \mathrm{c}$. & & & $100 \%$ & $100 \%$ & \\
\hline $\begin{array}{l}\text { Hyun (1997) } \\
(29)\end{array}$ & 62 & $0 \%$ & 3 c. $(5 \%)$ & $98 \%$ & $100 \%$ & a 3-107 m \\
\hline $\begin{array}{l}\text { Wei (1998) } \\
\text { (30) }\end{array}$ & 12 & $8 \%$ & $1-2(16 \%)$ & & $100 \%$ & a $24-36 \mathrm{~m}$ \\
\hline $\begin{array}{l}\text { Kojima (1999) } \\
\text { (31) }\end{array}$ & 54 & $0 \%$ & $5(9 \%)$ & $100 \%$ & & 9 y $50 m$ \\
\hline Hizawa (2000) & 51 c. 14 Q & $0 \%$ & $?$ & & & \\
\hline $\begin{array}{l}\text { Souto (2002) } \\
\text { (33) }\end{array}$ & 15 & $0 \%$ & $(7 \%)$ & $100 \%$ & & \\
\hline $\begin{array}{l}\text { Hunt (2002) } \\
\text { (34) }\end{array}$ & 45 & $0 \%$ & $6(13 \%)$ & & & \\
\hline $\begin{array}{l}\text { Waxman (2002) } \\
(35)\end{array}$ & 28 & $0 \%$ & $(0 \%)$ & $93 \%$ & $100 \%$ & a 2-74 m \\
\hline Sun (2002) & 16 & $0 \%$ & $?$ & $100 \%$ & & $12-17 m$ \\
\hline $\begin{array}{l}\text { Varas (2003) } \\
(37)\end{array}$ & 22 & $0 \%$ & $2(4,5 \%)$ & $91 \%$ & $100 \%$ & \\
\hline $\begin{array}{l}\text { Wehrmann (2004) } \\
\text { (38) }\end{array}$ & 20 & $0 \%$ & & $95 \%$ & $100 \%$ & $12 \mathrm{~m}$ \\
\hline Martínez-Ares (05) & 50 & $0 \%$ & $2(4 \%)$ & $98 \%$ & & \\
\hline Varas (2006) & 27 & $0 \%$ & $2(7,4 \%)$ & $92 \%$ & $100 \%$ & \\
\hline Resumen & +350 & $0-8 \%$ & $4-16 \%(10 \%)$ & $91-100 \%$ & $100 \%$ & \\
\hline
\end{tabular}

con resecciones completas que van del 91 al 100\%, y sin recidivas a mediano y largo plazo.

Nosotros sólo hemos tenido dos hemorragias $(7,4 \%)$, una autolimitada $(3,7 \%)$.

En el caso de los tumores carcinoides se acepta que deben ser menores de $1 \mathrm{~cm}$ (40), y localizados en la muscularis mucosa y/o en la submucosa, para que la tumorectomía sea curativa (40-44) (Tabla V).

La mayoría de trabajos con estos parámetros consiguen resecciones completas cercanas al $100 \%$. Los porcentajes de perforación y hemorragia son más variados.

Ono y cols. (42) comparan 14 tumores rectales resecados con bandas (resección completa del 100\%) con otros 14 casos en los que se aplicó polipectomía, y los márgenes estaban afectados en el $42 \%$.

Kobayashi y cols. (44) en un último estudio sobre 66 tumores carcinoides rectales afirman que en los casos en que el tamaño era menor de $10 \mathrm{~mm}$ (57 casos) no existía infiltración ni metástasis, mientras que los que eran mayores o iguales a $11 \mathrm{~mm}$ ( 9 casos) en 5 había infiltración de la muscular propia y en 4 metástasis.

En el manejo del cáncer esofagogástrico y colorrectal, la única manera de mejorar el pronóstico y la supervivencia es detectar el cáncer digestivo en fase precoz (cáncer superficial limitado a la mucosa) antes de la aparición de metástasis ganglionares.

De los distintos tratamientos locales sobre el cáncer superficial y la displasia de alto grado sólo la RME permite obtener una pieza de resección y disponer de un examen anatomopatológico completo. 
Tabla V. Tumorectomía de carcinoides. Resumen de la literatura

\begin{tabular}{|c|c|c|c|c|c|}
\hline Autor-año & $N^{\circ}$ & Perf. & $H D A$ & $R C$ & \\
\hline $\begin{array}{l}\text { Higaki (1997) } \\
(40)\end{array}$ & $18 \mathrm{c}$ & \multicolumn{4}{|c|}{ Rectales menores de $10 \mathrm{~mm}$} \\
\hline $\begin{array}{l}\text { Yohikane (1998) } \\
\text { (41) }\end{array}$ & $7 c$ & $1(14 \%)$ & $0(0 \%)$ & $100 \%$ & $6-46 m$ \\
\hline Ono (2003) & $14 \mathrm{c}$ & Bandas & & $100 \%$ & \\
\hline (42) & $14 \mathrm{c}$ & Polipectomía & & $58 \%$ & \\
\hline $\begin{array}{l}\text { Varas }(2003) \\
(37)\end{array}$ & $11 \mathrm{c}$ & P y una banda & $0(0 \%)$ & $100 \%$ & \\
\hline $\begin{array}{l}\text { Martínez-Ares (04) } \\
\text { (43) }\end{array}$ & $24 c$. & $\mathrm{x}: 9 \mathrm{~mm}$ & $1(4 \%)$ & $100 \%$ & \\
\hline Kobayashi (2005) & $66 c$ & \multirow{2}{*}{\multicolumn{4}{|c|}{$\begin{array}{l}\text { Rectales } \\
\text { Menores de } 10 \mathrm{~mm} \\
\text { Mayores de } 11 \mathrm{~mm}, 5 \text { infiltración MP y } 4 \text { metástasis }\end{array}$}} \\
\hline (44) & $\begin{array}{l}57 c . \\
9 c .\end{array}$ & & & & \\
\hline Resumen & $154 \mathrm{c}$ & $0-14 \%$ & $0-4-26 \%$ & $100 \%$ & \\
\hline
\end{tabular}

Perf.: perforación; HDA: hemorragia digestiva alta; RC: remisión completa.

Tabla VI. Mucosectomía. Revisión de la literatura

\begin{tabular}{|c|c|c|c|c|}
\hline Autor-año & $N^{\circ}$ & $R$ & M & C \\
\hline $\begin{array}{l}\text { Makuuchi (1996) } \\
\text { (45) }\end{array}$ & 255 c. esofágicos & $2,4 \%$ & $0,4 \%$ & - \\
\hline $\begin{array}{l}\text { Inoue (1998) } \\
(46)\end{array}$ & 142 c. esofágicos & & $0 \%$ & 0,7\% Perf. \\
\hline $\begin{array}{l}\text { Takekoshi (1995) } \\
\text { (47) }\end{array}$ & 308 c. gástricos & & & $3 \%$ \\
\hline $\begin{array}{l}\text { Kida (1998) } \\
\text { (48) }\end{array}$ & 246 c. gástricos & 2,8 & $0 \%$ & \\
\hline $\begin{array}{l}\text { Kojima (1998) R } \\
\text { (49) }\end{array}$ & 1832 c. gástricos & 2,6 & $1 \%$ & $\begin{array}{l}\text { 1,4 HDA } \\
\text { 0,5 Perf. }\end{array}$ \\
\hline $\begin{array}{l}\text { Inoue (1998) } \\
(46)\end{array}$ & 102 c. gástricos & 1,9 & $0 \%$ & \\
\hline $\begin{array}{l}\text { Giovannini (1999) } \\
(50)\end{array}$ & 64 c. (E y G) & 3.4 & & $12,7 \%$ \\
\hline $\begin{array}{l}\text { Ono }(2001) \\
(51)\end{array}$ & 479 c. gástricos & 2,0 & $0 \%$ & $5 \%$ \\
\hline $\begin{array}{l}\text { Adachi (2002) R } \\
\text { (52) }\end{array}$ & R gástricos (10 años) & & & \\
\hline $\begin{array}{l}\text { Oda (2005) R } \\
\text { (53) }\end{array}$ & \multicolumn{3}{|c|}{1.033 c. gástricos (98\% de resección) } & $\begin{array}{l}4 \% \text { Perf. } \\
13 \% \mathrm{HDA}\end{array}$ \\
\hline $\begin{array}{l}\text { Jung }(2005) \\
(54)\end{array}$ & 332 с. $91 \%$ & 1,9 & & \\
\hline $\begin{array}{l}\text { Ahmad (2002) } \\
(55)\end{array}$ & 101 c. varias lesiones & $0(27 \mathrm{~m})$ & & $21 \% \mathrm{HDA}$ \\
\hline $\begin{array}{l}\text { Su (2005) } \\
(56)\end{array}$ & 152 c. varias lesiones $c$ & olon & & $1,3 \% \mathrm{HDA}$ \\
\hline $\begin{array}{l}\text { Bories (2006) } \\
(57)\end{array}$ & 52 c. colon & & & $9,6 \%$ \\
\hline $\begin{array}{l}\text { Miquel (2006) } \\
(60)\end{array}$ & 9/18 c. digestivos & & $0 \%$ & $0 \%$ \\
\hline Resumen & & $2-4 \%$ & $0-1 \%$ & $1 \%$ Perf. \\
\hline
\end{tabular}

R: recidiva; M: mortalidad; C: complicaciones; Perf.: perforación; HDA: hemorragia digestiva alta.

La recurrencia local oscila entre el 2-4\%, con una supervivencia del $99 \%$, y con escasas complicaciones (perforación y hemorragia) que oscilan entre el 1-21\% para la hemorragia, siendo la perforación menor del 1\% (45-60) (Tabla VI).

En nuestros seis casos no hemos tenido complicaciones y la supervivencia ha sido del $100 \%$, sin recidivas. Un estudio multicéntrico de mayor número de casos ha sido recientemente publicado en nuestro país (60).

En Japón se ha empezado a sustituir la RME por la disección mucosa endoscópica (DME) con mayor tasa de resección en bloque, menos recidivas, menos complicaciones (hemorrágicas), pero con una tasa de perforación algo más elevada $(61,62)$.

\section{BIBLIOGRAFÍA}

1. Romero R, Pellicer F. Otras aplicaciones terapéuticas de la ultrasonografía endoscópica. Rev Esp Ecogr Dig 2001; 3: 72-8.

2. Varas MJ, Maluenda MD, Barturen A, et al. Ultrasonografía endoscópica intervencionista. Gastroenterol y Hepatol 2001; 24: 77-83.

3. Fernández-Urién I, Súbtil Iñigo JC, Muñoz-Navas M. Ecoendoscopia. Estado actual. Rev Esp Enferm Dig 2003; 95: 68-93.

4. Vila Costas JJ. Ultrasonografía endoscópica sectorial: situación actual e indicaciones. Rev Esp Enferm Dig 2005; 97: 899-906.

5. Vázquez-Sequeiros E, Norton ID, Clain JE, Wang KK, Affi A, Allen M, et al. Impact of EUS-guided fine-needle aspiration on lymph node staging in patients with esophageal carcinoma. Gastrointest Endosc 2001; 53: 751-7.

6. Varas MJ, Maluenda MD, Pou JM, et al. Valor de la ultrasonografía endoscópica en el estudio de los tumores submucosos del tracto digestivo. Gastroenterol y Hepatol 1998; 21: 121-4.

7. Giovannini M, Seitz JF, Monges G, Perrier H, Rabbia I. Fine needle aspiration cytology guided by endoscopic ultrasonography: results in 141 patients. Endoscopy 1995; 27: 171-7.

8. Wiersema MJ, Vilmann P, Giovannini M, Chang KJ, Wiersema LM. Endosonography-guided fine-needle aspiration biopsy: Diagnostic accuracy and complication assessment. Gastroenterology 1997; 112: 1087-95.

9. Chang KJ, Durbin TE, Katz KD, Lin F, Wuerker R. The clinical value of endoscopic ultrasound guided fine-needle aspiration in gastrointestinal and pulmonary malignancies. Am J Gastroenterol 1994; 89: A1704.

10. Gress FG, Hawes RH, Savides TJ, Ikenberry SO, Lehman GA. Endoscopic ultrasound-guided fine needle aspiration biopsy using linear array and radial scanning endosonography. Gastrointest Endosc 1997; 45: 243-50.

11. Matsui M, Goto H, Niwa Y, et al. Preliminary results of fine needle aspiration biopsy histology in upper gastrointestinal submucosal tumours. Endoscopy 1998; 30: 750-5.

12. Gu M, Ghafari S, Nguyen PT, Lin F. Cytologic diagnosis of gastrointestinal stromal tumors of the stomach by endoscopic ultrasound-guided fine-needle aspiration biopsy: Cytomorphological and immunohistochemical study of 12 cases. Diagn Cytopathol 2001; 25: 343-50.

13. Ando N, Goto H, Niwa Y, Hirooka Y, Ohmiya N, et al. The diagnosis of GI stromal tumors with EUS-guided fine needle aspiration with immunohistochemical análisis. Gastrointest Endosc 2002; 55: 37-43.

14. Dong Q, McKee G, Pitman M, Geisinger K, Tambouret R. Epithelioid variant of gastrointestinal stromal tumor: Diagnosis by fine-needle aspiration. Diagn Cytopathol 2003; 29: 55-60.

15. Kinoshita K, Isozaki K, Tsutsui S, Kitamura S, Hiraoka S, Watabe $\mathrm{K}$, et al. Endoscopic ultrasonography-guided fine needle aspiration biopsy in follow-up patients with gastrointestinal stromal tumours. Eur J Gastroenterol Hepatol 2003; 15: 1189-93.

16. Arantes V, Logroño R, Faruqi S, Ahmed I, Waxman I, Bhután MS Endoscopic sonographically guided fine-needle aspiration yield in submucosal tumors of the gastrointestinal tract. J Ultrasound Med 2004; 23: 1141-50.

17. Varas Lorenzo MJ, Gómez Rubio M, Souto Ruzo J. Características 
ecoendoscópicas de los tumores submucosos (TSM). Actitud a seguir y algoritmo. Rev Esp Enferm Dig 2004; 96: 215-8.

18. Harada N, Wiersema MJ, Wierserma LM. Endosonography guided fine-needle aspiration biopsy (EUS-FNA) in the evaluation of lymphadenopathy: staging accuracy of EUS FNA versus EUS alone. Gastrointest Endosc 1997; 45: AB31.

19. Williams DB, Sahai AV, Aabakken L, Penman ID, van Velse A, et al. Endoscopic ultrasound guided fine needle aspiration biopsy: a large single centro experience. Gut 1999; 44: 720-6.

20. Fritscher-Raven A, Sriam PVJ, Bobrowski C, et al. Mediastinal lymphadenopathy in patients with or without previous malignancy: EUS-FNA-based differential cytodiagnosis in 153 patients. Am J Gastroenterol 2000; 95: 2278-84.

21. Chen VK, Eloubeidi MA. Endoscopic ultrasound-guided fine-needle aspiration is superior to lymph node echofeatures: a prospective evaluation of mediastinal and peri-intestinal lymphadenopathy. Am J Gastroenterol 2004; 99: 628-33.

22. Chang KJ, Wiersema MJ, Giovannini M, Vilmann P, Erickson RA. A multi-center collaborative study on endoscopic ultrasound (EUS) guided fine-needle aspiration (FNA) of the pancreas. Gastrointest Endosc 1996; 43: 417-22.

23. Fritscher-Ravens A, Brand L, Knofel WT, et al. Comparison of endoscopic ultrasound-guided fine needle aspiration for focal pancreatic lesions in patients with normal parenchyma and chronic pancreatitis. Am J Gastroenterol 2002; 97: 2768-75.

24. Eloubeidi MA, Jhala D, Chhieng DC, Chen VE, Eltoum I, Vickers S et al. Yield of endoscopic ultrasound-guided fine-needle aspiration biopsy in patients with suspected pancreatic carcinoma. Cancer 2003; 99: 285-92.

25. O'Toole D, Palazzo L, Arotcarena R. Assessment of complications of EUS-guided fine needle aspiration. Gastrointest Endosc 2001; 53: 470-4.

26. Barawi M, Gottlieb K, Cunha B, Portis M, Gress F. A prospective evaluation of the incidence of bacteremia associated with EUS-guided fine-needle aspiration. Gastrointest Endosc 2001; 53: 189-92.

27. Bournet B, Migueres I, Delacroix M, et al. Early morbidity of endoscopic ultrasound: 13 years' experience at a referral center. Endoscopy 2006; 38: 349-54.

28. Kawamoto K, Yamada Y, Furukawa N, et al. Endoscopic submucosal tumorectomy for gastrointestinal submucosal tumors restricted to the submucosa: a new form of endoscopic minimal surgery. Gastrointest Endosc 1997; 46: 311-7.

29. Hyun JH, Jeen YT, Chun HJ, et al. Endoscopic resection of submucosal tumor of the esophagus: results in 62 patients. Endoscopy 1997; 29: $165-70$

30. Wei SC, Wong JM, Shieh MJ, Sun CT, Wang CY, Wang TH. Endoscopic resection of gastrointestinal submucosal tumors. Hepatogastroenterology 1998; 45: 114-8.

31. Kojima T, Takahashi H, Parra-Blanco A, Kohsen K, Fujita R. Diagnosis of submucosal tumor of the upper GI tract by endoscopic resection. Gastrointest Endosc 1999; 50: 516-22.

32. Hizawa K, Matsumoto T, Kouzuki T, Suekane H, Esaki M, Fujishima M. Cystic submucosal tumors in the gastrointestinal tract: endosonographic findings and endoscopic removal. Endoscopy 2000; 32: 712-4.

33. Souto J, Martínez-Ares D, Yánez JA, Vázquez Iglesias JL. Implicaciones terapéuticas de la ultrasonografía endoscópica en la patología tumoral. En: Souto J, edior. Ultrasonografía endoscópica. Tomo II. Barcelona: Ed. Glosa; 2002. p. 76-86.

34. Hunt G, Smith P, Faigel D. Yield of tissue sampling for submucosal lesions evaluated by EUS. Gastrointest Endosc 2002; 56: S102.

35. Waxman I, Saitoh Y, Raju GS, Watari J, Yokota K. High-frequency probe EUS-assisted endoscopic mucosal resection: a therapeutic strategy for submucosal tumors of the GI tract. Gastrointest Endosc 2002; 55: 44-9.

36. Sun S, Wang M, Sun S. Use of endoscopic ultrasound-guided injection in endoscopic resection of solid submucosal tumors. Endoscopy 2002; 34: 82-5.

37. Varas MJ, Espinós JC, Abad R, Bargalló D, Miquel JM. Resección endoscópica de tumores submucosos asistida por ecoendoscopia. Gastroenterol y Hepatol 2003; 26 (2): 108.

38. Wehrmann T, Martchenko K, Nakamura M, Riphaus A, Stergiou N. Endoscopic resection of submucosal esophageal tumors: A prospec- tive case series. Endoscopy 2004; 36: 802-7.

39. Martínez-Ares D, Varas MJ, Souto J, Espinós JC, Yañez J, Abad R, et al. Endoscopic resection gastrointestinal submucosal tumors assisted by endoscopic ultrasonography. Surg Endoscopy 2005; 19: 854-8.

40. Higaki S, Nishiaki M, Mitani N, et al. Effectiveness of local endoscopic resection of rectal carcinoids tumors. Endoscopy 1997; 29: 171-5.

41. Yohikane H, Goto H, Niwa Y, et al. Endoscopic resection of smal duodenal carcinoid tumors with strip biopsy technique. Gastrointest Endosc 1998; 47: 466-70.

42. Ono A, Fujii T, Saito Y, et al. Endoscopic submucosal resection of rectal carcinoid tumors with ligation device. Gastrointest Endosc 2003; 57: 583-7.

43. Martínez-Ares D, Souto J, Varas MJ, Espinós JC, Yánez J, Abad R et al. Resección endoscópica asistida por ecoendoscopia de tumores carcinoides del aparato digestivo. Rev Esp Enferm Dig 2004; 96 : 847-55.

44. Kobayashi K, Katsumata T, Yoshizawa S, et al. Indications of endoscopic polypectomy for rectal carcinoid tumors and clinical usefulness of endoscopic ultrasonography. Dis Colon and Rectum 2005; 48: 285-91.

45. Makuuchi H, Shimada H, Mizutani K, et al. Analisis of long term follow up patients after endoscopic mucosal resection for early esophageal cancer- with special reference to metachronous multiple cancers. Stomach and Intestine 1996; 31: 1223-33.

46. Inoue H. Endoscopic mucosal resection for esophageal and gastric mucosal cancers. Endoscopy 1998; 12: 355-9.

47. Takekoshi T, Baba Y, Ota H. Endoscopic resection of early gastric carcinoma: results of a retrospective analysis of 308 cases. Endoscopy $1995 ; 25: 352-8$.

48. Kida M, Tanabe S, Watanabe M, Kokutou M, Kondou I, et al. Staging of gastric cancer with endoscopic ultrasonography and endoscopic mucosal resection. Endoscopy 1998; 30: A64-A68.

49. Kojima T, Parra-Blanco A, Takahashi H, Fujita R. Outcome of endoscopic mucosal resection for early gastric cancer: review of the Japanese literature. Gastrointest Endosc 1998; 48: 550-5.

50. Giovannini M, Bernardini D, Monges G, et al. Mucosectomie endoscopique: facteurs pronostiques. Resultats chez 64 patients. Endoscopy 1999; 31: A20-P38.

51. Ono H, Kondo H, Gotoda T, et al. Endoscopic mucosal resection for treatment of early gastric cancer. Gut 2001; 48: 225-9.

52. Adachi Y, Shiraishi N, Kitano S. Modern treatment of early gastric cancer: review of Japanese experience. Dig Surg 2002; 19: 333-9.

53. Oda I, Gotoda T, Hamanaka I, et al. Endoscopic submucosal dissection for early gastric cancer: technical feasibility, operation time and complications from a large consecutive series. Dig Endosc 2005; 17 : 54-8.

54. Jung H, Lee G, Choi K, et al. Long-term outcome after endoscopic mucosal resection for early gastric cancer in Korea. Gut 2005; 54 (Supl. VII): A27.

55. Ahmad NA, Kochman ML, Long WB, Furth EE, Ginsberg GG. Efficacy, safety, and clinical outcomes of endoscopic mucosal resection: a study of 101 cases. Gastrointest Endosc 2002; 55: 390-6

56. Su MY, Hsu CM, Ho YP, et al. Endoscopic mucosal resection for colonic non-polypoid neoplastic lesions. Am J Gastroenterol 2005; 100: 2174-9.

57. Bories E, Pesenti C, Monges G, Lelong B, Moutardier V, Delpero JR, et al. Endoscopic mucosal resection for advanced sessile adenoma and early-stage colorectal carcinoma. Endoscopy 2006; 38: 231-5.

58. Lightdale CJ. Endoscopic mucosal resection: This is our turf. Endoscopy 2004; 36: 808-10.

59. Wong RF, Bhutani MS. Therapeutic endoscopy and endoscopic ultrasound for gastrointestinal malignancies. Expert Rev Anticancer Ther 2005; 5: 705-18.

60. Miquel JM, Abad R, Souto J, et al. Mucosectomía guiada por USE en el cáncer digestivo. Rev Esp Enferm Dig 2006; 98: 591-6.

61. Kakushima N, Yahagi N, Fujishiro M, et al. Efficacy and safety of endoscopic submucosal dissection for tumors of the esophagogastric junction. Endoscopy 2006; 38: 170-4.

62. Polkowski M. Endoscopic diagnosis and treatment of upper gastrointestinal tumors. Endoscopy 2006; 38: 1070-5. 